\title{
Decay of False Vacua via Impurities in String Theories
}

\section{Yutaka Ookouchi*}

Faculty of Arts and Science, Kyushu University, Fukuoka 819-0395, Japan

E-mail: vutaka.ookouchidartsci.kyushu-u.ac.jp

\section{Yuichiro Nakai}

Department of Physics and Astronomy, Rutgers University, Piscataway, NJ 08854, USA

E-mail: vnakaiephysics.rutgers.edu

\section{Norihiro Tanahashi}

Institute of Mathematics for Industry, Kyushu University, Fukuoka 819-0395, Japan

E-mail: tanahashieimi.kvushu-u.ac.jp

\begin{abstract}
We investigate catalysis induced by a dyonic impurity in the metastable vacuum studied by Kachru, Pearson and Verlinde, which can be relevant to vacuum decay in the KKLT scenario. The impurity is a D3-brane wrapping on $\mathbb{S}^{3}$ in the Klebanov-Strassler geometry. The effect of the D3-brane can be encoded in the world-volume theory of an NS5-brane as an electromagnetic field on it. As the field strength becomes large, instability of the vacuum enhances. As a result, the lifetime of the metastable vacuum becomes drastically shorter.
\end{abstract}

Corfu Summer Institute 2018 "School and Workshops on Elementary Particle Physics and Gravity" (CORFU2018)

31 August - 28 September, 2018

Corfu, Greece

\footnotetext{
* Speaker.
} 


\section{Introduction}

Recent progress in string theories has been revealing that there exist a large number of metastable vacua. This involved vacuum structure of string theories is called string landscape [四]. Among such metastable vacua, if there is a vacuum corresponding to our universe, it has to have a small positive cosmological constant. In the celebrated work [వ], Kachru, Kallosh, Linde and Trivedi (KKLT) proposed a scenario realizing de Sitter vacua in string theories. Since the KKLT vacuum is metastable, it decays within a finite time-length. There are two kinds of instabilities to the KKLT vacuum. One is destabilization of the volume-moduli and the other is annihilation of $\overline{\mathrm{D}}$-branes with background fluxes: To uplift the anti-de Sitter vacuum, KKLT added $\overline{\mathrm{D} 3}$-branes at the tip of the deformed conifold [[]], and such anti-branes can decay with the background fluxes [B]]. In this paper, we discuss a catalytic effect for the latter decay process due to an impurity in the KKLT setup. Since the decay process occurs quite near the tip of the conifold, we can treat the total geometry as the non-compact Klebanov-Strassler (KS) geometry [团] without losing control. This allows us to neglect gravitational effects in the four-dimensional spacetime and thus drastically simplify the analysis of the vacuum lifetime. In this non-compact limit, the system is essentially the same as the model studied by Kachru, Pearson and Verlinde (KPV) [3]. See ref. [5] for an early work of a decay process of the KPV vacuum. Our goal is to examine the consequence of the D3-brane impurity and its catalytic effect in the KKLT scenario, for which we need to take into account nontrivial electromagnetic fields on the brane in the setup of [B] as we explain below.

As we explain in section \, In our setup, the D3-brane impurity behaves as a dyonic particle in the four-dimensional Minkowski spacetime. This kind of solitonic object was firstly discussed in [G] and later studied in various setups of string theories [ $[$ ]. In this paper, we investigate further on stringy metastable solitons and show that their existence makes the lifetime of the metastable vacuum drastically shorter. The impurity enhances the bubble nucleation rate and causes a spatially inhomogeneous decay of the vacuum.

The idea of catalysis induced by solitons was firstly pointed out in ref. [8] and applied to phenomenological model building later [ [Q, [0]]. Also, it was discussed in the context of string theory in ref. $[$ [ $]$. In this paper, we would like to go a step further to a more involved but quite interesting setup in string theory such as the KKLT model. The analysis of [3] works almost as is even in the KKLT model, a difference from [B] is that we need to take into account the nontrivial electric and magnetic fields induced on the NS5-brane due to the D3-brane impurity. We will study how these fields affect the tunneling rate of the metastable vacuum by employing the thin-wall approximation and numeric analysis.

The plan of the work is following. In section 2, we briefly review the geometry near the tip of the deformed conifold [ [ 9 ] and the KPV metastable vacuum realized on it. Then we numerically show dyonic solutions in the KPV metastable vacuum, which corresponds to the field configuration before the bubble nucleation. In section 3, we study the catalytic effect induced by such dyonic objects. By using the thin-wall approximation of the solutions, we show that the lifetime of the vacuum becomes drastically shorter. Section 4 is devoted to discussions and conclusions. Interested readers should refer to [ㅁ] for details of the analysis. 


\section{A dyonic solution in the KPV metastable vacuum}

The decay process proposed by KPV is the phase transition from the metastable vacuum of the KKLT model to the supersymmetric true vacuum induced by annihilation of $\overline{\mathrm{D} 3}$-branes and background flux [B]. The KKLT model is realized on the deformed conifold background [䧃], whose tip is round off at the radius $r^{2}=\frac{3}{2^{5 / 3}} \varepsilon^{4 / 3} e^{2 \tau / 3}$ due to the background flux. The metric of the nonvanishing sub-manifold at the tip $\tau=0$ is given by [四, 미]

$$
d s_{4+3}^{2}=b_{0}^{2} g_{s} M \alpha^{\prime}\left[\eta_{\mu v} d \tilde{x}^{\mu} d \tilde{x}^{\nu}+d \Psi^{2}+\sin ^{2} \Psi d \Omega_{2}^{2}\right]
$$

where $\tilde{x}^{\mu} \equiv \frac{a_{0}}{\sqrt{b_{0}^{2} g_{s} M \alpha^{\prime}}} x^{\mu}(\mu=0,1,2,3)$ are the dimensionless coordinates in the four-dimensional Minkowski spacetime part of the geometry. Here, $a_{0}^{2} \equiv \frac{\varepsilon^{4 / 3}}{2^{1 / 3} c_{0}^{1 / 2}{ }_{s s} M \alpha^{\prime}} \simeq 0.9366 \frac{\varepsilon^{4 / 3}}{g_{s} M \alpha^{\prime}}, b_{0}^{2} \equiv \frac{2}{6^{1 / 3}} c_{0}^{1 / 2} \simeq$ 0.9326 are constants defined in terms of $c_{0} \simeq 0.7180$, the string coupling constant $g_{s}$, the background flux $M=\frac{1}{4 \pi \alpha^{\prime}} \int_{\mathbb{S}^{3}} F_{3}$ and the deformation parameter $\varepsilon$. As was discussed in the paper [B]], the $\overline{\mathrm{D} 3}$-branes added to this background puff up by the Myers effect [113] and make an NS5-brane

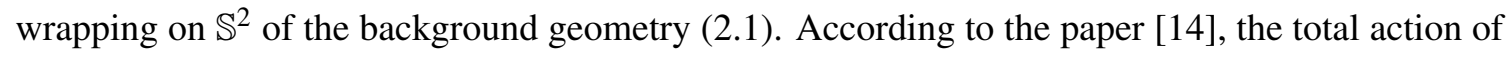
the NS5-brane is given by

$$
S=-\frac{T_{N S}}{g_{s}^{2}} \int d^{6} \xi \sqrt{-\operatorname{det}\left(g_{a b}+2 \pi g_{s} \alpha^{\prime} \widetilde{\mathscr{F}}\right)}-T_{N S} \int B_{6},
$$

where $T_{N S}$ is the tension of the NS5-brane and $2 \pi \alpha^{\prime} \widetilde{\mathscr{F}}=2 \pi \alpha^{\prime} F_{2}-C_{2}$. Then the decay of KPV metastable vacuum is given as a tunneling process for the NS5-brane from the metastable vacuum to the supersymmetric vacuum (see figure $\mathbb{W}$ ).

Now, we introduce an impurity by wrapping a D3-brane on $\mathbb{S}^{3}$ at $\tau=0$. Since the RR 3-form flux threads $\mathbb{S}^{3}$, a charge of the fundamental string is induced on the wrapped D3-brane [피]. To reconcile the charge conservation for the induced charge, we have to introduce the fundamental string ending on the D3-brane and the NS5-brane. This object can be seen as a dyonic particle from the viewpoint of Minkowski spacetime spanned by the NS5-brane (see figure 1 ). When the metastable vacuum decays in this setup, the D3-brane forms a bound state with the domain wall created by the decay as follows. At the domain wall, the NS5-brane sweeps a portion of $\mathbb{S}^{3}$ between the loci corresponding to the metastable vacuum and the true vacuum. Thus the domain wall NS5brane and the dyonic D3-brane are on the top of each other on $\mathbb{S}^{3}$. In this case, the D3-brane dissolves into the NS5-brane to form a bound state [16], and the effect of the D3-brane manifests in the Lagrangian as the electromagnetic field on the NS5-brane.

Now we are ready to consider the Lagrangian describing the NS5-brane. Let us discuss the electromagnetic field in the dimensionless coordinate $F_{\mu \nu} d x^{\mu} \wedge d x^{v}=\widetilde{F}_{\mu \nu} d \tilde{x}^{\mu} \wedge d \tilde{x}^{\nu}$. The diagonal block corresponding to Minkowski spacetime of the matrix $\tilde{g}_{a b}+2 \pi g_{s} \alpha^{\prime} \tilde{F}_{a b}$ can be represented as

$$
b_{0}^{2} g_{s} M \alpha^{\prime} \times\left(\begin{array}{cccc}
\left(-1+\dot{\Psi}^{2}\right) & \dot{\Psi} \Psi^{\prime}+\mathscr{E} & 0 & 0 \\
\dot{\Psi} \Psi^{\prime}-\mathscr{E} & \left(1+\Psi^{\prime 2}\right) & 0 & 0 \\
0 & 0 & \tilde{r}^{2} & \mathscr{B} \\
0 & 0 & -\mathscr{B} & \tilde{r}^{2} \sin ^{2} \theta
\end{array}\right)
$$




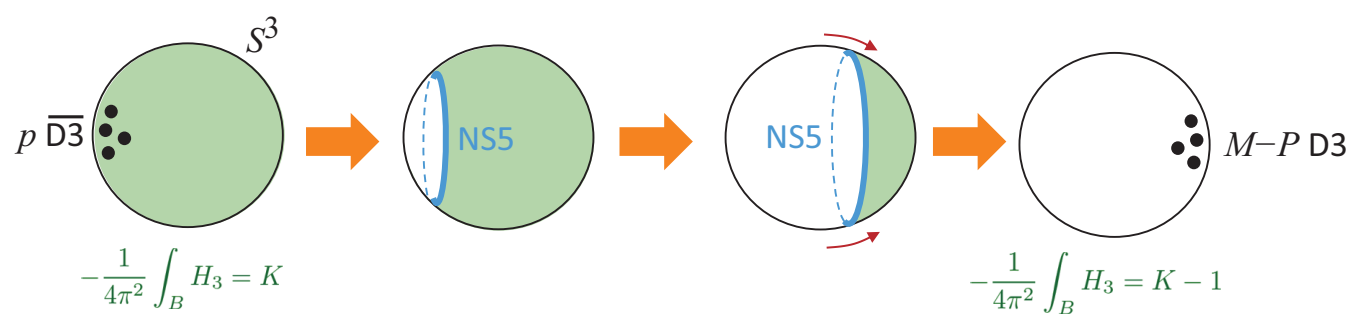

Figure 1: A schematic of the KPV vacuum decay. $\overline{\mathrm{D} 3}$-branes added to the deformed conifold background form a NS5-brane that resides at the metastable point at $\Psi \sim 2 \pi p / b_{0}^{4} M$. This vacuum decays to the supersymmetric true vacuum corresponding to $\Psi=\pi$ by reducing the background $H_{3}$ flux by one unit.

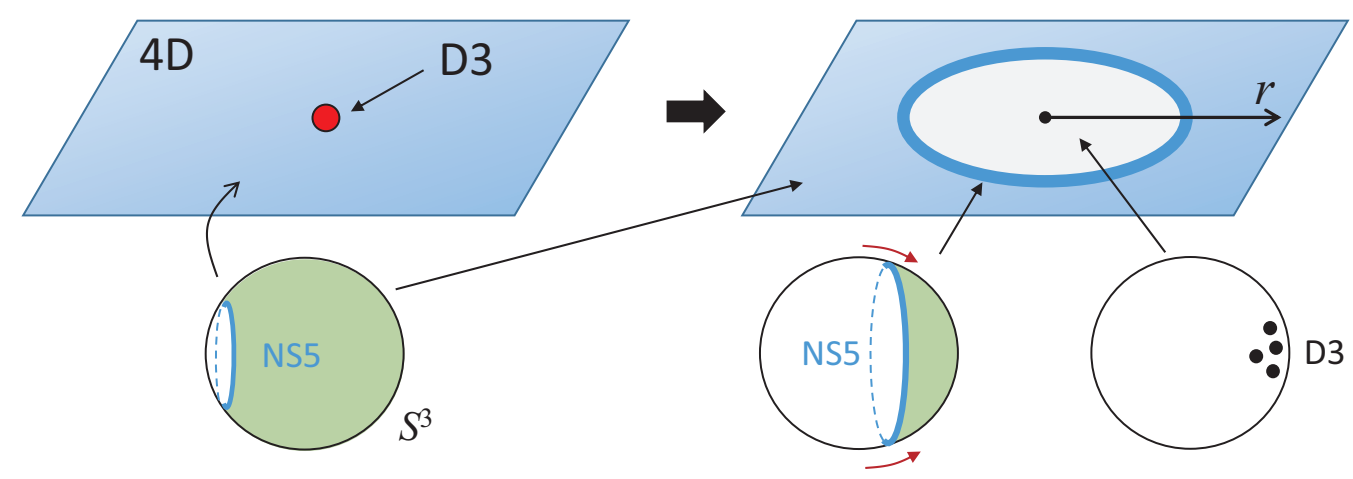

Figure 2: The D3-brane impurity behaves as a dyonic particle in the four-dimensional Minkowski spacetime spanned by the NS5-brane. In the KPV vacuum decay the D3-brane dissolves into the domain wall made of the NS5-brane to form a bound state.

where $\tilde{r}$ is the dimensionless radial coordinate, and the NS5-brane is wrapping $\mathbb{S}^{2}$ at $\Psi=\Psi(\tilde{t}, \tilde{r})$. The dot and prime denote $\tilde{t}$ and $\tilde{r}$ derivatives, respectively. $\mathscr{B} \equiv \frac{2 \pi g_{s} \alpha^{\prime}}{a_{0}^{2}} B$ stands for the dimensionless magnetic field multiplied by $\tilde{r}^{2}$, which is a constant and proportional to the number of the D3-branes added as the impurity. $\mathscr{E} \equiv \frac{2 \pi g_{s} \alpha^{\prime}}{a_{0}^{2}} E$ is the electric field induced on the brane. Also the diagonal block of the matrix $\tilde{g}_{a b}+2 \pi g_{s} \alpha^{\prime} \tilde{F}_{a b}$ corresponding to the internal space $d \Omega_{2}^{2}$ in (L.T) spanned by $\theta_{I}, \phi_{I}$ is given by

$$
\left(\begin{array}{cc}
b_{0}^{2} g_{s} M \alpha^{\prime} \sin ^{2} \Psi & \alpha^{\prime}\left(\pi g_{s} p-g_{s} M\left(\Psi-\frac{1}{2} \sin 2 \Psi\right)\right) \sin \theta_{I} \\
-\alpha^{\prime}\left(\pi g_{s} p-g_{s} M\left(\Psi-\frac{1}{2} \sin 2 \Psi\right)\right) \sin \theta_{I} & b_{0}^{2} g_{s} M \alpha^{\prime} \sin ^{2} \Psi \sin ^{2} \theta_{I}
\end{array}\right)
$$

where $p$ is the number of the anti-D3-branes. Thus, the DBI action for the NS5-brane $S_{\mathrm{DBI}}=$ $-\frac{T_{N S}}{g_{s}^{2}} \int d^{6} \xi \sqrt{-\operatorname{det}\left(g_{a b}+2 \pi g_{s} \alpha^{\prime} \widetilde{\mathscr{F}}\right)}=-16 \pi^{3} g_{s} M^{3} b_{0}^{4} \mu_{5} \widetilde{S}_{\mathrm{DBI}}$ is given by

$$
\widetilde{S}_{\mathrm{DBI}}=\int d \tilde{t} d \tilde{r} \sqrt{1-\dot{\Psi}^{2}+\Psi^{\prime 2}-\mathscr{E}^{2}} \sqrt{\tilde{r}^{4}+\mathscr{B}^{2}} \frac{1}{\pi} \sqrt{b_{0}^{4} \sin ^{4} \Psi+\left(\frac{\pi p}{M}-\left(\Psi-\frac{1}{2} \sin 2 \Psi\right)\right)^{2}}
$$

where we defined $\mu_{5}=T_{N S} \alpha^{\prime 3}$. Also the Chern-Simons term in (2.2]) is written as, using the KS 
solution [田],

$$
S_{\mathrm{CS}}=-T_{N S} \int B_{6}=\frac{\mu_{5}}{g_{s} \alpha^{\prime 3}} \int(d V)_{4} \int_{S^{2}} C_{2}=16 \pi^{2} g_{s} M^{3} b_{0}^{4} \mu_{5} \int d \tilde{r} d \tilde{t} \tilde{r}^{2}\left(\Psi-\frac{1}{2} \sin 2 \Psi\right)
$$

In total, the action is given by

$$
\begin{aligned}
& S=16 \pi^{3} g_{s} M^{3} b_{0}^{4} \mu_{5} \widetilde{S}=16 \pi^{3} g_{s} M^{3} b_{0}^{4} \mu_{5} \int d \tilde{t} d \tilde{r} \widetilde{\mathscr{L}} \\
& \widetilde{\mathscr{L}}=-V_{2}(\Psi) \sqrt{1-\dot{\Psi}^{2}+\Psi^{\prime 2}-\mathscr{E}^{2}} \sqrt{\tilde{r}^{4}+\mathscr{B}^{2}}+\frac{\tilde{r}^{2}}{\pi}\left(\Psi-\frac{1}{2} \sin 2 \Psi\right) .
\end{aligned}
$$

Here we defined $V_{2}(\Psi)$ by

$$
V_{2}(\Psi)=\frac{1}{\pi} \sqrt{b_{0}^{4} \sin ^{4} \Psi+\left(\frac{\pi p}{M}-\left(\Psi-\frac{1}{2} \sin 2 \Psi\right)\right)^{2}}
$$

$\mathscr{E}$ depends on $\tilde{r}$, hence it is convenient to change the variable that is independent of $\tilde{r}$. This can be accomplished by the Legendre transformation in terms of the electric displacement $\mathscr{D} \equiv \frac{\partial \tilde{\mathscr{L}}}{\partial \mathscr{E}}=$

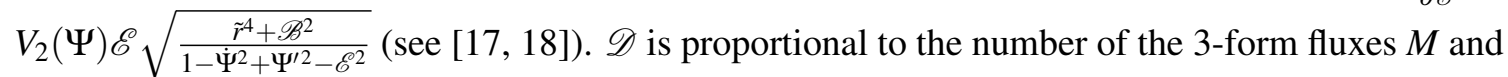
that of the D3-branes added as the impurity. Then the new Lagrangian which is a function of $\mathscr{B}$ and $\mathscr{D}$ is given by

$$
\widetilde{\mathscr{L}_{D}}=\widetilde{\mathscr{L}}-\mathscr{D} \mathscr{E}=-\sqrt{V_{2}(\Psi)^{2}\left(\tilde{r}^{4}+\mathscr{B}^{2}\right)+\mathscr{D}^{2}} \sqrt{1-\dot{\Psi}^{2}+\Psi^{\prime 2}}+\frac{\tilde{r}^{2}}{\pi}\left(\Psi-\frac{1}{2} \sin 2 \Psi\right)
$$

To understand basic features of the KPV metastable vacuum and its decay, let us consider the action with $\mathscr{B}=\mathscr{D}=0$. In this case, we can rewrite the action as

$$
\widetilde{S}=\frac{1}{4 \pi} \int d \tilde{r} d \tilde{t} d \theta d \phi \tilde{r}^{2} \sin \theta\left[-V_{2}(\Psi) \sqrt{1-\dot{\Psi}^{2}+\Psi^{\prime 2}}+\frac{1}{\pi}\left(\Psi-\frac{1}{2} \sin 2 \Psi\right)\right] .
$$

Once we put $\Psi^{\prime}=0$, this action coincides with that for a homogeneous configuration shown in [B]]. From this action, the potential energy for a static configuration is given by

$$
\widetilde{V}(\mathscr{B}=\mathscr{D}=0) \propto V_{2}(\Psi)-\frac{1}{\pi}\left(\Psi-\frac{1}{2} \sin 2 \Psi\right) .
$$

As shown in figure B, this potential has a true vacuum at $\Psi=\pi$ and a metastable point at $\Psi \sim$ $2 \pi p / b_{0}^{4} M[B]$. The tunneling process proceeds from the metastable vacuum $\Psi \sim 2 \pi p / b_{0}^{4} M$ to the supersymmetric true vacuum $\Psi=\pi$.

Now let us study inhomogeneous $\left(\Psi^{\prime} \neq 0\right)$ static solutions with $\mathscr{E}, \mathscr{B} \neq 0$ imposing the regularity at the center $\Psi^{\prime}(\tilde{r}=0)=0$ following [ए8] . These solutions correspond to the metastable configuration before the tunneling process occurs. In figure 4 , we show numerical solutions of such static configurations obtained using the relaxation method. At large $\tilde{r}$, contributions of $\mathscr{D}$ and $\mathscr{B}$ become negligible, and then the NS5-brane position converges to the KPV metastable point. As the radius becomes small, the NS5-brane position $\Psi$ becomes closer to $\Psi=\pi$ for a purely magnetic 


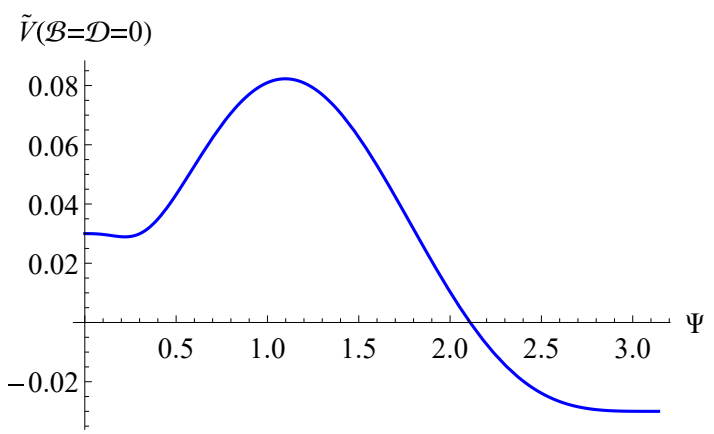

Figure 3: Potential energy for a static NS5-brane with $\mathscr{B}=\mathscr{D}=0$. Besides the global potential minimum is at $\Psi=\pi$, there is a local minimum at $\Psi=0.220$, which is approximately given by $\Psi=2 \pi p / b_{0}^{4} M$ when $p / M \ll 1[$ [3] .
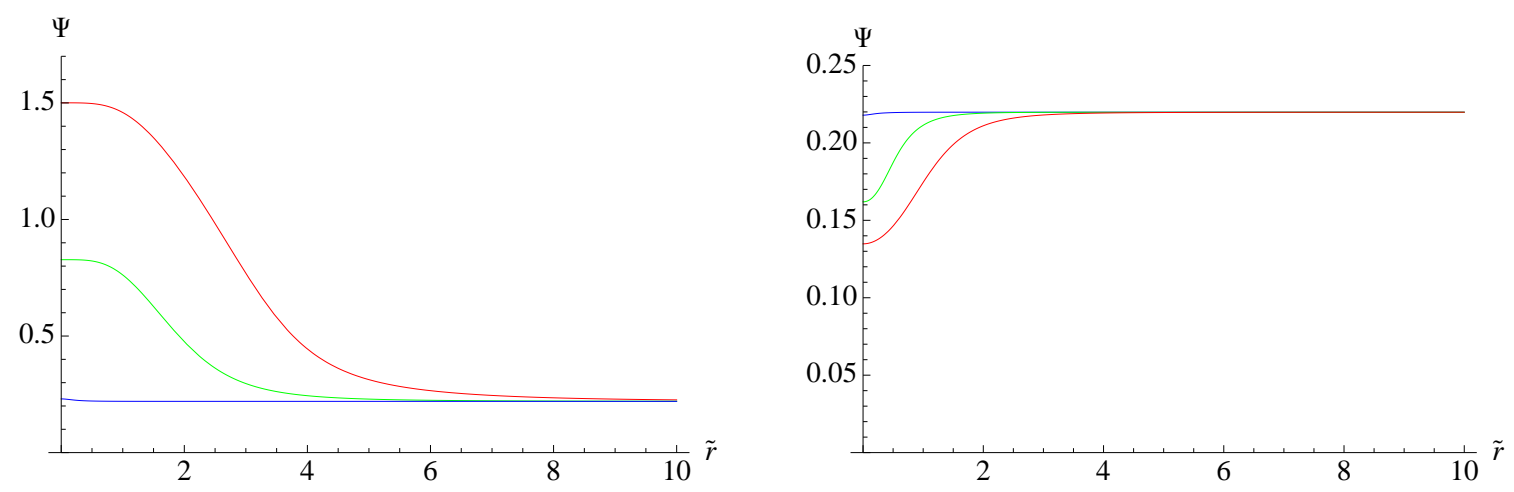

Figure 4: Plots of static solutions for $p / M=0.03$. The right panel shows purely magnetic solutions with $\mathscr{D}=0$, where the red, green and blue lines correspond to $\mathscr{B}=1.4,0.3$ and 0.001 . The left panel shows the purely electric solutions with $\mathscr{B}=0$ where the red, green and blue lines correspond to $\mathscr{D}=1,0.3$ and 0.001 .

solutions while it becomes closer to $\Psi=0$ for a purely electric solutions. This behavior can be understood as follows. Expanding the action to the leading order in $\mathscr{D}$ and $\mathscr{B}$, we find

$$
\widetilde{S} \supset 2 \pi \int d^{4} \tilde{x}\left[V_{2}(\Psi)\left(\frac{\mathscr{B}}{4 \pi \tilde{r}^{2}}\right)^{2}+\frac{1}{V_{2}(\Psi)}\left(\frac{\mathscr{D}^{2}}{4 \pi \tilde{r}^{2}}\right)^{2}\right],
$$

where we recovered the angular integrals. Let us focus on the purely magnetic case first, for which only the first term is present in the above. We see that the energy density due to nonzero $\mathscr{B}$ becomes larger as $\tilde{r}$ becomes smaller. To compensate this energy increase, $\Psi$ is forced to shift to smaller value since $V_{2}(\Psi)$, defined by ([.5)), is an increasing function of $\Psi$. On the other hand, in the purely electric case, $V_{2}(\Psi)$ appears as the denominator of the second term, hence $\Psi$ becomes larger as $\tilde{r}$ becomes smaller to make the total energy smaller.

It is worth noting that in the present setup, the 3-form flux $M$ is a large number because the curvature of the conifold should be large enough to make the supergravity approximation reliable. When we wrap the D3 brane on the $\mathbb{S}^{3}, M$ units of the fundamental charge are induced on it, which means that $\mathscr{D}$ is proportional to $M$. Therefore, in our assumption, $\mathscr{D}$ is larger than $\mathscr{B}$. In this case, 
from figure 4 , we expect that profile functions of the dyonic particles should be much similar to the ones in the left panel of the figure. For this type of dyonic solutions, $\Psi$ is shifted to larger value around the center. This feature would enhance the phase transition from the metastable vacuum since the configuration of the NS5-brane is pushed toward that of the true vacuum $\Psi=\pi$. In the next section, we will confirm that the tunneling rate to the true vacuum is indeed enhanced when nonzero $\mathscr{B}$ and $\mathscr{D}$ are present.

\section{Numerical study of the decay rate}

We studied static configurations of the NS5-branes in the previous section and observed that the branes are bent near the origin of $\Psi$ when a dyonic impurity exists at the origin. In this section, by assuming the existence of a stable solution for an appropriate choice of the parameters $\mathscr{B}, \mathscr{D}$, we estimate the decay rate using the thin-wall approximation to the bubble.

\subsection{Thin-wall approximation}

The goal of this section is to construct solutions describing the phase transition from the metastable KPV vacuum to the true vacuum. An obstacle for it is that the Lagrangian (2.6) depends on both $\tilde{t}$ and $\tilde{r}$, hence one would need to solve two-dimensional partial differential equations to obtain such solutions. To simplify this problem we employ the thin-shell approximation for the domain wall, with which the profile of a domain wall is given by $\Psi=\left(\Psi_{\max }-\right.$ $\left.\Psi_{\min }\right)[1-\theta(\tilde{r}-R(\tilde{t}))]+\Psi_{\min }$. where $\theta$ is the step function. The NS5-brane annihilates with the background flux at the bubble wall, hence the electromagnetic field on the NS5-brane becomes zero inside the bubble. Namely, we set $\mathscr{B}=\mathscr{D}=0$ for $\tilde{r}<R$. For this ansatz, the differentials of $\Psi(\tilde{t}, \tilde{r})$ become $\frac{\partial \Psi}{\partial \tilde{r}}=-\left(\Psi_{\max }-\Psi_{\min }\right) \delta(\tilde{r}-R(\tilde{t})), \frac{\partial \Psi}{\partial \tilde{t}}=\left(\Psi_{\max }-\Psi_{\min }\right) \dot{R}(\tilde{t}) \delta(\tilde{r}-R(\tilde{t}))$, and then the kinetic part of the Lagrangian (2.6) is approximated as $\sqrt{1-\dot{\Psi}^{2}+\Psi^{\prime 2}} \simeq\left(\Psi_{\max }-\right.$ $\left.\Psi_{\min }\right) \sqrt{1-\dot{R}^{2}} \delta(\tilde{r}-R(\tilde{t}))$. Applying the above approximations to (R.J), the action

$$
\begin{aligned}
& \widetilde{S}^{\mathrm{tot}}=\int d \tilde{t}\left[\int_{0}^{R} d \tilde{r}\left(V_{2}\left(\Psi_{\min }\right) \sqrt{\tilde{r}^{4}+\mathscr{B}^{2}+\frac{\mathscr{D}^{2}}{V_{2}^{2}\left(\Psi_{\min }\right)}}-V_{2}\left(\Psi_{\max }\right) \tilde{r}^{2}\right)+\frac{R^{3}}{3 \pi}\left(\Psi_{\max }-\frac{\sin 2 \Psi_{\text {max }}}{2}\right)\right. \\
& \left.-\frac{R^{3}}{3 \pi}\left(\Psi_{\min }-\frac{\sin 2 \Psi_{\min }}{2}\right)+\int_{\Psi_{\text {max }}}^{\Psi_{\text {min }}} d \Psi\left(\sqrt{V_{2}(\Psi)^{2}\left(R^{4}+\mathscr{B}^{2}\right)+\mathscr{D}^{2}} \sqrt{1-\dot{R}^{2}}-\sqrt{V_{2}(\Psi)^{2} \mathscr{B}^{2}+\mathscr{D}^{2}}\right)\right] .
\end{aligned}
$$

To make this total action finite, we subtracted the action for the static solution $R(\tilde{\tau})=0$.

To see the physical meaning of (B. $(\mathbb{C})$, let us study the potential energy of a static configuration

$$
\begin{aligned}
4 \pi \widetilde{V}^{\mathrm{tot}}= & -\Delta V \frac{4 \pi R^{3}}{3}-4 \pi V_{2}\left(\Psi_{\min }\right) \int_{0}^{R} d \tilde{r}\left(\sqrt{\tilde{r}^{4}+\mathscr{B}^{2}+\frac{\mathscr{D}^{2}}{V_{2}^{2}\left(\Psi_{\min }\right)}}-\tilde{r}^{2}\right) \\
& +4 \pi \int_{\Psi_{\min }}^{\Psi_{\max }} d \Psi\left(\sqrt{V_{2}(\Psi)^{2}\left(R^{4}+\mathscr{B}^{2}\right)+\mathscr{D}^{2}}-\sqrt{V_{2}(\Psi)^{2} \mathscr{B}^{2}+\mathscr{D}^{2}}\right),
\end{aligned}
$$

where we defined $\Delta V=\mathscr{V}\left(\Psi_{\min }\right)-\mathscr{V}\left(\Psi_{\max }\right)$ with $\mathscr{V}(\Psi)=\frac{1}{4 \pi}\left[V_{2}(\Psi)-\frac{1}{\pi}\left(\Psi-\frac{1}{2} \sin 2 \Psi\right)\right]$. The first term is the energy deficit due to the true vacuum inside the bubble. The second term is the 
energy deficit due to disappearance of the electromagnetic fields inside the bubble. The third term corresponds to the surface energy of the bubble originating from the tension and electromagnetic fields on it.

\subsection{Bounce action}

Now, we are ready to study a catalytic decay of the KPV metastable vacuum. To estimate the decay rate, we use Coleman's method [ए9] and proceed basically along the lines of [ए7]. Let us first introduce functions defined by

$$
\begin{aligned}
T(R, \mathscr{B}, \mathscr{D}) \equiv & -\int_{\Psi_{\max }}^{\Psi_{\min }} d \Psi \sqrt{V_{2}(\Psi)^{2}\left(R^{4}+\mathscr{B}^{2}\right)+\mathscr{D}^{2}} \\
H(R, \mathscr{B}, \mathscr{D}) \equiv & \frac{R^{3}}{3 \pi}\left(\Psi_{\max }-\Psi_{\min }-\frac{1}{2} \sin 2 \Psi_{\max }+\frac{1}{2} \sin 2 \Psi_{\min }\right)+T(R=0, \mathscr{B}, \mathscr{D}) \\
& -V_{2}\left(\Psi_{\max }\right) \int_{0}^{R} d \tilde{r} \tilde{r}^{2}+V_{2}\left(\Psi_{\min }\right) \int_{0}^{R} d \tilde{r} \sqrt{\tilde{r}^{4}+\mathscr{B}^{2}+\frac{\mathscr{D}^{2}}{V_{2}\left(\Psi_{\min }\right)^{2}}}
\end{aligned}
$$

With these functions, the Euclidean action can be written as

$$
\widetilde{S}_{E}^{\text {tot }}=\int d \tilde{\tau}\left[-H(R, \mathscr{B}, \mathscr{D})+T(R, \mathscr{B}, \mathscr{D}) \sqrt{1+\dot{R}^{2}}\right] .
$$

When $p / M$ is small, which is necessary for neglecting the back-reaction of the $\overline{\mathrm{D} 3}$-branes, the angular coordinate at the metastable vacuum is approximately given by $\Psi_{\min }=2 \pi p / b_{0}^{4} M$ [B]]. As for the maximum value of $\Psi$, we simply assume the value for the supersymmetric vacuum, namely $\Psi_{\text {max }}=\pi$. Below, we show the dimensionless effective potential defined by $\widetilde{V}=-H(R, \mathscr{B}, \mathscr{D})+$ $T(R, \mathscr{B}, \mathscr{D})$ for several values of $\mathscr{B}$ and $\mathscr{D}$. In figure [1, we choose $\mathscr{D}=0, p / M=0.08$. The blue, green and red lines correspond to $\mathscr{B}=0.01,0.3$ and 1 respectively. From the right panel of the figure $\mathbb{1}$, one sees that there exists a metastable point at nonzero $R$. We denote this minimum $R_{\text {ini }}$. The effective potential for the case $\mathscr{B}=0, \mathscr{D} \neq 0$ show similar features.
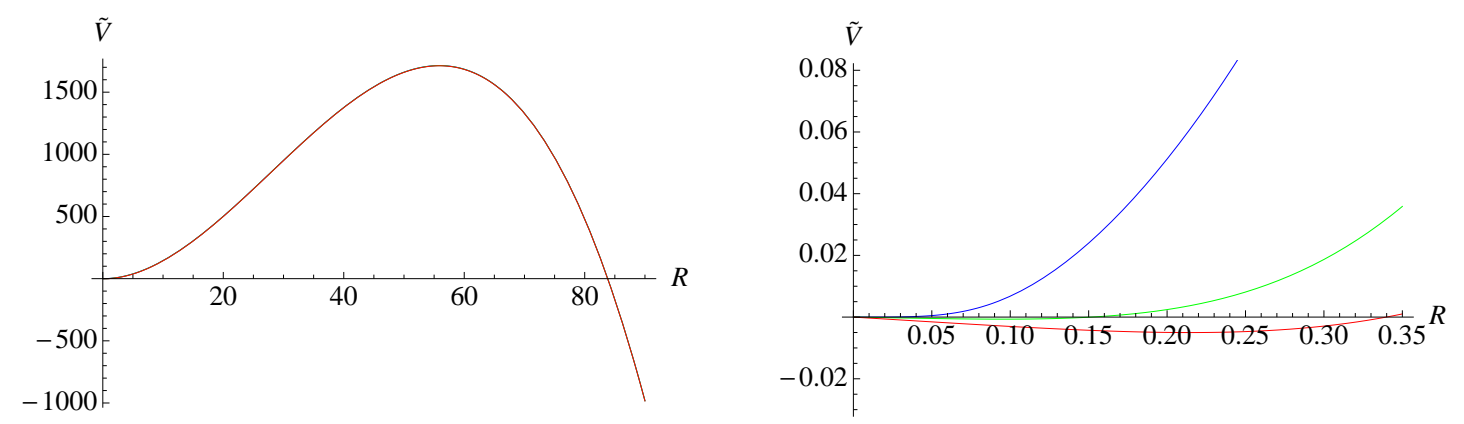

Figure 5: Plots of the dimensionless potential $\widetilde{V}$ with $p / M=0.08$. We choose $\mathscr{D}=0$ and the blue, green and red lines correspond to $\mathscr{B}=0.01,0.3$ and 1 respectively. In the right panel, we magnify the functions around $R=0.15$. Each curve has a local minimum at $R=R_{\text {ini }}>0$, at which the domain wall before the vacuum decay resides.

By using the initial condition given by $R=R_{\text {ini }}$ and $\dot{R}=0$ at $\tilde{\tau}=0$, the conserved Hamiltonian can be represented as $\frac{T(R, \mathscr{B}, \mathscr{D})}{\sqrt{1+\dot{R}^{2}}}=H(R, \mathscr{B}, \mathscr{D})-H\left(R_{\text {ini }}, \mathscr{B}, \mathscr{D}\right)+T\left(R_{\text {ini }}, \mathscr{B}, \mathscr{D}\right)$, which can be used 
to eliminate $\dot{R}$ from the bounce action. Also, to obtain the bounce action we subtract the action for the static solution $R=R_{\text {ini }}$

$$
\begin{aligned}
\frac{1}{2} \widetilde{B}_{b}=\widetilde{S}_{E}-\widetilde{S}_{E}\left(R_{\mathrm{ini}}\right) & =\int d \tau\left[-H(R, \mathscr{B}, \mathscr{D})+T(R, \mathscr{B}, \mathscr{D}) \sqrt{1+\dot{R}^{2}}-K_{0}\right] \\
& =\int_{R_{\mathrm{ini}}}^{R_{*}} d R \sqrt{T(R, \mathscr{B}, \mathscr{D})^{2}-\left(H(R, \mathscr{B}, \mathscr{D})+K_{0}\right)^{2}},
\end{aligned}
$$

where we introduced $K_{0}=-H\left(R_{\text {ini }}, \mathscr{B}, \mathscr{D}\right)+T\left(R_{\text {ini }}, \mathscr{B}, \mathscr{D}\right)$.

In figure $\mathbb{6}$, we show numerical values of the bounce action (B.4) and its dependence on $\mathscr{B}$ and $\mathscr{D}$. The main feature of the result is that the bounce action decreases as $\mathscr{B}$ or $\mathscr{D}$ increases. The effect of $\mathscr{B}$ and $\mathscr{D}$ is significant mainly in the small $\tilde{r}$ region, and in such a region the total action (B.3) depends on $\mathscr{B}$ and $\mathscr{D}$ through a combination $\sqrt{\mathscr{B}^{2}+\mathscr{D}^{2} / V_{2}^{2}\left(\Psi_{\min }\right)}$. This dependence would be the origin of behavior of the bounce action shown in figure 6 , where the bounce action decreases linearly with respect to $\mathscr{B}$ when $\mathscr{D}=0$ (left panel) while the behavior becomes more involved when both $\mathscr{B}$ and $\mathscr{D}$ are turned on (right panel of figure 6 ). The tunneling probability is an exponential of the bounce action, and hence we may conclude that nonzero $\mathscr{B}$ and $\mathscr{D}$ enhances the tunneling probability significantly. The $\mathscr{B}$ and $\mathscr{D}$ fields are nothing but the manifestation of the D3-brane impurities we introduced, and in this sense one concludes that the KPV vacuum is efficiently catalyzed by such impurities to decay to the true vacua.
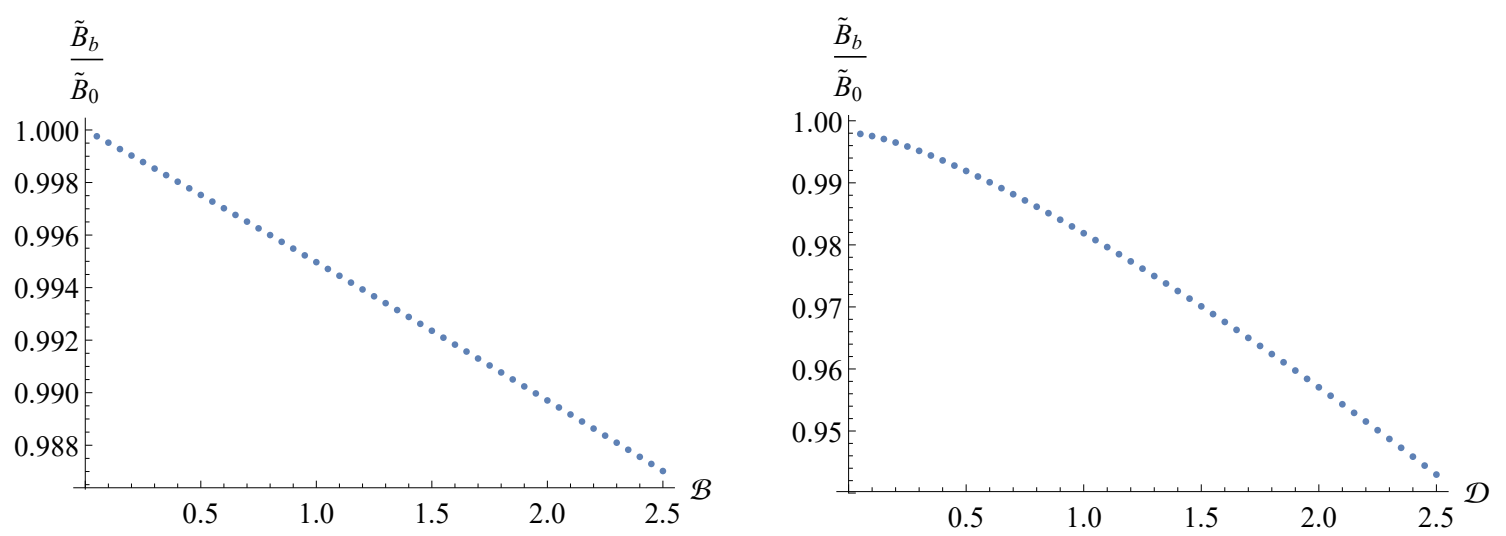

Figure 6: Numerical values of the bounce action for $p / M=0.08$ normalized by $\widetilde{B}_{0}$ which is the bounce action for $\mathscr{B}=\mathscr{D}=0$. In the left panel, we show the $\mathscr{B}$ dependence of the bounce action for $\mathscr{D}=0$. In the right panel, we show the $\mathscr{D}$ dependence when $\mathscr{B}=0.4$. In both cases, as $\mathscr{B}$ or $\mathscr{D}$ increases, the bounce action becomes smaller.

\section{Discussions and conclusions}

In this work, we focused on the decay of metastable vacua in Type IIB string theory and investigated the catalytic effect induced by D3-branes wrapped on $\mathbb{S}^{3}$ at the tip of the deformed conifold. We first studied the bound state of the D3-brane and domain wall NS5-brane which connects the metastable vacuum to true vacuum. We found that a dyonic particle induces instability of the metastable state near the particle. Then, we estimated the decay rate employing the Coleman's 
method and the thin-wall approximation. We showed that the life-time of the metastable vacuum becomes shorter when non-vanishing electromagnetic field is present. We also pointed out that this type of the vacuum decay may occur even in the de Sitter vacuum in the KKLT scenario if D3-brane impurities are present.

In this work we neglected the gravitational effects in the four-dimensional spacetime by taking the decoupling limit and focusing on the tip of the deformed conifold. It is desirable to improve our analysis taking the gravitational effect into account, so that we can study the influences of the vacuum decay discussed in this work to the de Sitter universe realized in the KKLT scenario. For example, catalysis of the phase transition due to the black holes and compact objects were discussed in ref. [ए] ] taking the gravitational effect into account. Though the "catalyst" in our setup is a stringy particle and qualitatively different from theirs, it would be fruitful to make connection between these catalytic processes to gain deeper insight into the phenomenology in the early universe and to find observational evidence of the background theory governing it.

This decay process is associated with a dyonically charged spherical domain wall, and it might be interesting to examine its observational signature in our universe. In some cases, such spherical domain walls collapse to form black holes. Studying the dynamics and observational consequences of such spherical domain walls employing techniques of, e.g., ref. [R] ] would be one of possible future directions of our study.

\section{Acknowledgement}

We are grateful to Minoru Eto for useful discussions. YN is supported by the DOE grant DE-SC0010008. YO would like to thank Rutgers University for their hospitality. YO and NT are supported by Grant-in-Aid for Scientific Research from the Ministry of Education, Culture, Sports, Science and Technology, Japan (No.17K05419, No.18H01214 and No.18K03623) and AY 2018 Qdai-jump Research Program of Kyushu University.

\section{References}

[1] R. Bousso and J. Polchinski, JHEP 0006, 006 (2000) [hep-th/0004134]; S. Kachru, R. Kallosh, A. D. Linde and S. P. Trivedi, Phys. Rev. D 68, 046005 (2003) [hep-th/0301240]; L. Susskind, In Carr, Bernard (ed.): Universe or multiverse? 247-266 [hep-th/0302219]; S. Ashok and M. R. Douglas, JHEP 0401, 060 (2004) [hep-th/0307049].

[2] S. Kachru, R. Kallosh, A. D. Linde and S. P. Trivedi, Phys. Rev. D 68, 046005 (2003) [hep-th/0301240]; S. Kachru, R. Kallosh, A. D. Linde, J. M. Maldacena, L. P. McAllister and S. P. Trivedi, JCAP 0310, 013 (2003) [hep-th/0308055].

[3] S. Kachru, J. Pearson and H. L. Verlinde, JHEP 0206, 021 (2002) [hep-th/0112197].

[4] I. R. Klebanov and M. J. Strassler, JHEP 0008, 052 (2000) [hep-th/0007191].

[5] A. R. Frey, M. Lippert and B. Williams, Phys. Rev. D 68, 046008 (2003) [hep-th/0305018].

[6] H. Verlinde, hep-th/0611069.

[7] A. Kasai and Y. Ookouchi, Phys. Rev. D 91, no. 12, 126002 (2015) [arXiv:1502.01544 [hep-th]]; JHEP 1506, 098 (2015) [arXiv:1504.00479 [hep-th]]; A. Kasai, Y. Nakai and Y. Ookouchi, JHEP 
1606, 029 (2016) [arXiv:1508.04608 [hep-th]]; Y. Nakai and Y. Ookouchi, Phys. Lett. B 762, 321 (2016) [arXiv:1608.01232 [hep-th]].

[8] P. J. Steinhardt, Nucl. Phys. B 190, 583 (1981); Phys. Rev. D 24, 842 (1981); Y. Hosotani, Phys. Rev. D 27, 789 (1983); U. A. Yajnik, Phys. Rev. D 34, 1237 (1986).

[9] B. H. Lee, W. Lee, R. MacKenzie, M. B. Paranjape, U. A. Yajnik and D. h. Yeom, Phys. Rev. D 88, 085031 (2013) [arXiv:1308.3501 [hep-th]]; B. Kumar, M. B. Paranjape and U. A. Yajnik, Phys. Rev. D 82, 025022 (2010) [arXiv:1006.0693 [hep-th]]; B. Kumar and U. Yajnik, Nucl. Phys. B 831, 162 (2010) [arXiv:0908.3949 [hep-th]]; B. Kumar and U. A. Yajnik, Phys. Rev. D 79, 065001 (2009) [arXiv:0807.3254 [hep-th]].

[10] T. Hiramatsu, M. Eto, K. Kamada, T. Kobayashi and Y. Ookouchi, JHEP 1401, 165 (2014) [arXiv:1304.0623 [hep-ph]]; K. Kamada, T. Kobayashi, K. Ohashi and Y. Ookouchi, JHEP 1305, 091 (2013) [arXiv:1303.2740 [hep-ph]]; M. Eto, Y. Hamada, K. Kamada, T. Kobayashi, K. Ohashi and Y. Ookouchi, JHEP 1303, 159 (2013) [arXiv:1211.7237 [hep-th]].

[11] Y. Nakai, Y. Ookouchi and N. Tanahashi, arXiv:1808.10235 [hep-th].

[12] C. P. Herzog, I. R. Klebanov and P. Ouyang, hep-th/0108101.

[13] R. C. Myers, JHEP 9912, 022 (1999) [hep-th/9910053];

[14] E. Eyras, B. Janssen and Y. Lozano, Nucl. Phys. B 531, 275 (1998) [hep-th/9806169].

[15] E. Witten, JHEP 9807, 006 (1998) [hep-th/9805112]; T. Banks and N. Seiberg, Phys. Rev. D 83, 084019 (2011) [arXiv:1011.5120 [hep-th]]; M. Berasaluce-Gonzalez, P. G. Camara, F. Marchesano and A. M. Uranga, JHEP 1304, 138 (2013) [arXiv:1211.5317 [hep-th]].

[16] J. Polchinski, “String Theory 1,2," Cambridge University Press (1998); B. Zwiebach, Cambridge University Press (2009).

[17] R. Emparan, Phys. Lett. B 423, 71 (1998) [hep-th/9711106]; K. Hashimoto, JHEP 0207, 035 (2002) [hep-th/0204203]; Y. Hyakutake, JHEP 0105, 013 (2001) [hep-th/0103146]; D. K. Park, S. Tamarian, Y. G. Miao and H. J. W. Muller-Kirsten, Nucl. Phys. B 606, 84 (2001) [hep-th/0011116].

[18] I. R. Klebanov and S. S. Pufu, JHEP 1108, 035 (2011) [arXiv:1006.3587 [hep-th]].

[19] S. R. Coleman, Phys. Rev. D 15, 2929 (1977) [Erratum-ibid. D 16, 1248 (1977)].

[20] R. Gregory, I. G. Moss and B. Withers, JHEP 1403, 081 (2014) [arXiv:1401.0017 [hep-th]]; P. Burda, R. Gregory and I. Moss, JHEP 1508, 114 (2015) [arXiv:1503.07331 [hep-th]]; N. Oshita and J. Yokoyama, arXiv:1601.03929 [gr-qc]; N. Oshita, M. Yamada and M. Yamaguchi, arXiv:1808.01382 [gr-qc]; R. Gregory, K. M. Marshall, F. Michel and I. G. Moss, arXiv:1808.02305 [hep-th].

[21] K. i. Maeda, Gen. Rel. Grav. 18, 931 (1986); N. Tanahashi and C. M. Yoo, Class. Quant. Grav. 32, no. 15, 155003 (2015) [arXiv:1411.7479 [gr-qc]]; H. Deng, J. Garriga and A. Vilenkin, JCAP 1704, no. 04, 050 (2017) [arXiv:1612.03753 [gr-qc]]; H. Deng and A. Vilenkin, JCAP 1712, no. 12, 044 (2017) [arXiv:1710.02865 [gr-qc]]; H. Deng, A. Vilenkin and M. Yamada, arXiv:1804.10059 [gr-qc]. 